PITHA 02/06

hep-ph/0202056

\title{
Exclusive non-leptonic $B$ meson decays from QCD
}

\author{
M. Beneke $e^{a *}$

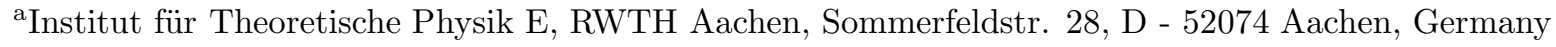 \\ A brief report on the QCD factorization approach to exclusive $B$ decays and its applications is presented. In \\ an appendix a subtle issue concerning partial integration in the parameterization of infrared-sensitive power \\ corrections is clarified.
}

\section{INTRODUCTION}

Exclusive non-leptonic decays play a crucial part in the on-going programme to clarify the sources of $\mathrm{CP}$ violation and rare flavour-changing phenomena. As regards CP violation this is illustrated by the fact that there exists a fair knowledge of the length of each of the sides of the unitarity triangle from semi-leptonic $B$ decays and $B \bar{B}$ mixing. Since recently, the angle $\beta$ (more precisely, $\sin 2 \beta$ ) is determined fairly accurately by the time-dependent $\mathrm{CP}$ asymmetry in the nonleptonic decay $B_{d} \rightarrow J / \psi K_{S}$ and related ones. However, the direct measurement of the other angle $\gamma$, the phase of $V_{u b}^{*}$ in the standard phase convention, remains a challenge to theory and experiment. Although $\gamma$ is determined indirectly through the other measurements, the complementary direct determination is important, since meson mixing and rare non-leptonic decays could be affected by new flavour-changing interactions.

The angle $\gamma$ can be determined from decays with some kind of interference of $b \rightarrow c \bar{u} D$ (no phase) and $b \rightarrow u \bar{c} D$ (phase $\gamma$ ) tree transitions and their conjugates $(D=d, s)$ or from decays with interference of $b \rightarrow u \bar{u} D$ (tree, phase $\gamma$ ) and $b \rightarrow D q \bar{q}$ (penguin, phase $0(D=s), \beta(D=d)$ ). There exist a few methods that allow one to obtain $\gamma$ in a theoretically clean way, which means that no calculation of strong interaction effects is needed, but these methods are all difficult to realize experimentally at present. The more general

\footnotetext{
*Talk presented at the 5th KEK Topical Conference "Frontiers in Flavour Physics", Tsukuba, Japan, November 2001
}

situation is that some information on the decay amplitudes is required to extract $\gamma$ from the interference term in branching fractions or CP asymmetries. In technical terms one needs to compute the matrix elements $\left\langle f\left|O_{i}\right| \bar{B}\right\rangle$, where $O_{i}$ is an operator in the weak effective Hamiltonian and $f$ is a particular exclusive final state.

\section{THEORY OF EXCLUSIVE DECAYS}

\subsection{QCD-improved factorization}

The QCD factorization approach [1.:2] uses heavy quark expansion methods $\left(m_{b} \gg \Lambda_{\mathrm{QCD}}\right)$ and soft-collinear factorization (particle energies $\gg \Lambda_{\mathrm{QCD}}$, "colour-transparency") to compute the matrix elements $\left\langle f\left|O_{i}\right| \bar{B}\right\rangle$ in an expansion in $1 / m_{b}$ and $\alpha_{s}$. Only the leading term in $1 / m_{b}$ assumes a simple form. The basic formula is

$$
\begin{aligned}
& \left\langle M_{1} M_{2}\left|O_{i}\right| \bar{B}\right\rangle=F^{B \rightarrow M_{1}}(0) \int_{0}^{1} d u T^{I}(u) \Phi_{M_{2}}(u) \\
& +\int d \xi d u d v T^{I I}(\xi, u, v) \Phi_{B}(\xi) \Phi_{M_{1}}(v) \Phi_{M_{2}}(u),(1)
\end{aligned}
$$

valid whenever $M_{2}$ is a light meson (or, at least in principle, a charmonium state) and $M_{1}$ any light or heavy meson. ( $M_{1}$ is the meson that picks up the spectator quark from the $B$ meson. If $M_{1}$ is heavy the second line is higher order in $1 / m_{b}$.) The formula shows that there is no long-distance interaction between the constituents of the meson $M_{2}$ and the $\left(B M_{1}\right)$ system at leading order in $1 / m_{b}$. This is the precise meaning of factorization. In particular, the hard scattering kernels $T^{I, I I}$ contain all rescattering phases at this order and include hard interactions with the specta- 
tor quark $\left(T^{I I}\right)$. The following non-perturbative inputs are needed: $F^{B \rightarrow M_{1}}$ - a $B \rightarrow M_{1}$ form factor; $\Phi_{M_{i}}$ - the standard light-cone distribution amplitudes for light mesons; $\Phi_{B}$ - the lightcone distribution amplitude of the $B$ meson [3[6], some aspects of which remain to be understood. The QCD factorization approach extends the Brodsky-Lepage approach to exclusive hard processes [7], because it shows factorization also when soft interactions dominate the $B \rightarrow M_{1}$ form factor, as is expected [8].

\subsection{Theory status}

Leading order in $1 / m_{b}$. Proofs of the factorization formula for heavy-light final states exist at two loops [2] and probably to all orders [9 10]. These proofs must be extended to charmless final states by including the hard-spectator interaction. In practical applications the calculations are currently done at next-to-leading order in perturbation theory. There exist theoretical uncertainties from input parameters, which can be estimated, the most important of which are $\left|V_{u b}\right|$ and form factors. The theory is presently complete for flavour non-singlet mesons.

Power corrections in $1 / m_{b}$. Factorization does not and is not expected to hold at subleading order. Attempts to compute subleading power corrections to hard spectator-scattering in perturbation theory usually result in infrared divergences, which signal the breakdown of factorization. A subset of power corrections has been parametrized or estimated in 2, 11, 12]. It is common practice in applications of perturbative QCD to not include errors from power corrections. One would wish to do better here, first because nonleptonic decays test fundamental aspects of weak interactions, second because some power corrections related to scalar currents are enhanced by factors such as $m_{\pi}^{2} /\left(\left(m_{u}+m_{d}\right) \Lambda_{\mathrm{QCD}}\right)$ [1]. At least these effects should be estimated and included into the error budget. This has been attempted in [12]. All weak annihilation contributions belong to this class of effects.

\subsection{Overview of applications}

QCD-improved factorization has been applied to date to the following final states:
- $P P$, where $P$ denotes a light pseudo-scalar non-singlet meson [1, 12 [15]. This is the application developed furthest.

- some $P V$ final states, where $V$ denotes a light vector meson 16, 17.

- some $V V$ final states [18]. (In the heavy quark limit both vector mesons are longitudinally polarized.)

- $D^{(*)} L$, where $L$ denotes a light pseudoscalar or vector meson 2, 19,20.

- $D^{(*)} X$, where $X$ denotes a scalar or "exotic" meson [21.

- $J / \psi K$ 22,23, $J / \psi K^{*} 24,25$. The factorization approach should apply in the limit $m_{c} \rightarrow \infty$, but one expects corrections to factorization of order $\Lambda_{\mathrm{QCD}} /\left(m_{c} \alpha_{s}\right) \sim 1$ [2].

- Radiative final states $V \gamma$ [26 28] and $V l^{+} l^{-}$ 26] including isospin-breaking effects that could cause a different rate for neutral and charged $B$ meson decay [29].

- New physics effects due to a modification of the $b \rightarrow s g$ transition [30] or due to R-parity violating supersymmetry [31].

Of these final states only $D^{(*)} L$ and $P P$ will be discussed below.

\subsection{Other approaches}

Naive factorization [32]. Naive factorization follows from the factorization formula for $D^{(*)} L$, $L_{1} L_{2}$ final states, when $\alpha_{s} \rightarrow 0$ and $m_{b} \rightarrow \infty$. QCD-improved factorization extends naive factorization in the same sense in which the QCDimproved parton model extends the naive parton model.

The "PQCD" approach [33]. In this approach the decay amplitude is calculated in the BrodskyLepage formalism. The main difference with the QCD-improved factorization approach arises through the stronger assumption that Sudakov suppression renders the $B \rightarrow M_{1}$ form factor and many power-suppressed effects (such as weak annihilation) calculable. The advocates of the QCD 
factorization approach do not agree with the proponents of the PQCD approach on conceptual aspects of the implementation of Sudakov suppression and on the claim that the result is perturbative and accurate [34].

\section{SELECTED RESULTS}

\section{1. $B \rightarrow D^{(*)} L$}

The phenomenology of these decays is discussed in [2]. QCD-improved factorization explains the experimentally well-established fact that the colour-allowed class-I decays to $D^{(*)+} L^{-}$ are controlled by an approximately universal coefficient $\left|a_{1}\right| \simeq 1.05$. On the other hand, factorization is not expected to hold for colour-suppressed class-II decays to $D^{(*) 0} L^{0}$. The corresponding coefficient $a_{2}$ is therefore depends on the final state. The precise nature of $a_{2}$ depends, however, on the counting scheme. If the $D$ meson is considered as heavy $\left(m_{c} / m_{b}\right.$ fixed, $\left.m_{b} \rightarrow \infty\right), x a_{2} / a_{1}$ is powersuppressed in the heavy quark limit. The power suppression is due to

$x \equiv \frac{\left(m_{B}^{2}-m_{\pi}^{2}\right) f_{D} F_{0}^{B \rightarrow \pi}\left(m_{D}^{2}\right)}{\left(m_{B}^{2}-m_{D}^{2}\right) f_{\pi} F_{0}^{B \rightarrow D}\left(m_{\pi}^{2}\right)}$,

but with $x \simeq 0.9$ this suppression is only formal. If the $D$ meson is considered as light $\left(m_{c}\right.$ fixed, $\left.m_{b} \rightarrow \infty\right), x a_{2} / a_{1}$ counts as order 1 and becomes calculable. (A crude estimate has been given in 22.) In this case one expects an important nonuniversal contribution from spectator-scattering. In both cases $x a_{2} / a_{1}$ should be smaller than 1 due to colour factors.
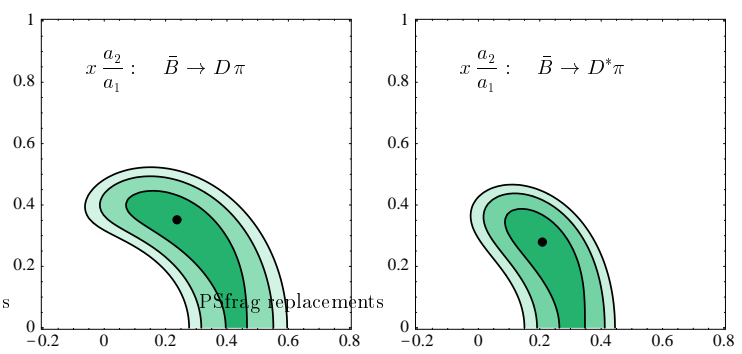

Figure 1. Determination of $x a_{2} / a_{1}$ for $D^{(*)} \pi$ final states [20].
The measurement of $\bar{B}^{0} \rightarrow D^{(*) 0} \pi^{0}$ allows one for the first time to construct the complete isospin triangle for $B \rightarrow D^{(*)} \pi$ decays and hence to determine the magnitude and rescattering phase of $x a_{2} / a_{1}$, see Figure 18. The result is that $\left|a_{2}\right| \simeq 0.4$ is sizeable for $\pi D$ final states. Together with $\left|a_{2}\right| \simeq 0.25$ for $K J / \psi$ final states this provides clear evidence for the non-universality of coloursuppressed decays, but it is difficult to interpret this result quantitatively since the theory is not expected to be accurate for $\pi^{0} D^{0}$ and $K J / \psi$ final states. For further discussion of this result from the perspective of QCD factorization see [20].

\section{2. $\gamma$ from $B \rightarrow \pi \pi, \pi K$}

The possibility to determine the CP-violating angle $\gamma$ by comparing the calculation of branching fractions into $\pi \pi$ and $\pi K$ final states with the corresponding data has been investigated in detail [12] (see also [15]). The branching fractions for the modes $B^{\mp} \rightarrow \pi^{\mp} \pi^{0}$ and $B^{\mp} \rightarrow \pi^{\mp} \bar{K}^{0}$, which depend only on a single weak phase to very good approximation, are well described by the theory. This demonstrates that the magnitude of the tree and penguin amplitude is obtained correctly, where for the penguin amplitude the 1-loop radiative correction is important to reach this conclusion. There is, however, a relatively large normalization uncertainty for the $\pi K$ final states, which are sensitive to weak annihilation and the strange quark mass through the scalar penguin amplitude. This uncertainty can be partially eliminated by taking ratios of branching fractions. The agreement is less good for branching fractions with significant interference of tree and penguin amplitudes, if $\gamma$ is assumed to take values around $55^{\circ}$ as favoured by indirect constraints. This may indicate an interesting alternative determination of $\gamma$, but the CP-averaged branching fractions sensitive to $\gamma$ also depend on the strong phase difference of the two interfering amplitudes. This dependence is suppressed as long as the phase difference is small since only its cosine enters the calculation. With some exceptions (such as the final state $\pi^{0} \pi^{0}$ ) QCDimproved factorization predicts that strong phase differences are not large, since they are parametrically of order $\alpha_{s}$ or $\Lambda_{\mathrm{QCD}} / m_{b}$, and it therefore 


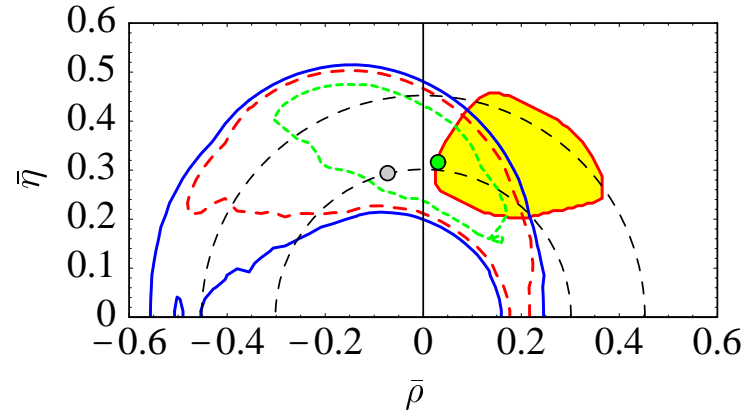

Figure 2. $\quad 95 \%$ (solid), 90\% (dashed) and $68 \%$ (short-dashed) confidence level contours in the $(\bar{\rho}, \bar{\eta})$ plane obtained from a global fit to the CP averaged $B \rightarrow \pi K, \pi \pi$ branching fractions, using the scanning method as described in 35. The right dot shows the overall best fit, whereas the left dot indicates the best fit for the default hadronic parameter set. The light-shaded region indicates the region preferred by the standard global fit [35], excluding the direct measurement of $\sin (2 \beta)$.

also predicts small direct $\mathrm{CP}$ asymmetries, up to $(10-15) \%$ (with a preference around $5 \%$ ) for the $\pi^{0} K^{ \pm}, \pi^{ \pm} K^{0}$ final states. The current data seem to favour small CP asymmetries, but they are not yet accurate enough to decide upon whether the QCD factorization approach allows one to predict strong phases quantitatively.

The current interpretation of charmless nonleptonic $B$ decays can be summarized by a global fit of the Wolfenstein parameters $\bar{\rho}, \bar{\eta}$ to the six CP averaged $\pi \pi, \pi K$ branching fractions shown in Figure 2. The result is consistent with the standard fit based on meson mixing and $\left|V_{u b}\right|$, but shows a preference for larger $\gamma$ or smaller $\left|V_{u b}\right|$. If the estimate of the theory uncertainty (included in the curves in the Figure) is correct, non-leptonic decays together with $\left|V_{u b}\right|$ from semi-leptonic decays already imply the existence of a CP-violating phase of $V_{u b}$ at the 2-3 $\sigma$ level.

\section{3. $\sin (2 \alpha)$ or $\gamma$ from $B_{d}(t) \rightarrow \pi^{+} \pi^{-}$}

The angle $\alpha$ (or $\gamma$, given the $B \bar{B}$ mixing phase), can be determined from the time-dependent $\mathrm{CP}$ asymmetry in $B_{d} \rightarrow \pi^{+} \pi^{-}$decay alone, if the rel-

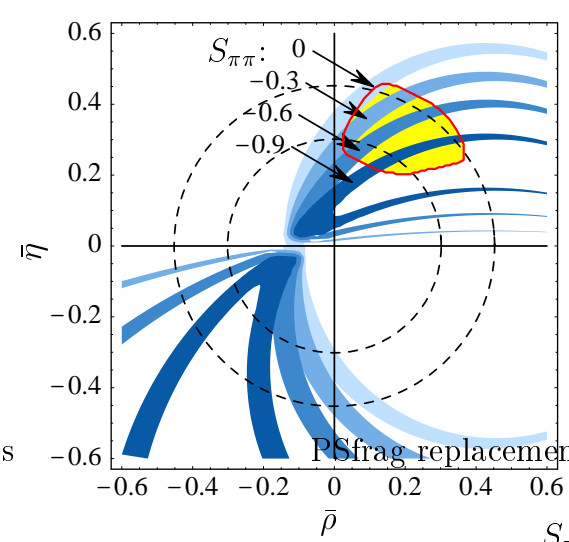

Figure 3. Constraint from $S_{\pi \pi}$ in the $(\bar{\rho}, \bar{\eta})$ plane.

ative magnitude of the penguin amplitude, $P / T$, can be computed. The asymmetry is given by

$$
\begin{aligned}
A_{\mathrm{CP}}[\pi \pi](t)= & S_{\pi \pi} \sin \left(\Delta M_{B_{d}} t\right) \\
& +A_{\mathrm{CP}}^{\mathrm{dir}}[\pi \pi] \cos \left(\Delta M_{B_{d}} t\right),
\end{aligned}
$$

where $S_{\pi \pi}=\sin (2 \alpha)$, if $P / T=0$, in which case the direct $\mathrm{CP}$ asymmetry $A_{\mathrm{CP}}^{\text {dir }}[\pi \pi]$ vanishes. The direct $\mathrm{CP}$ asymmetry is proportional to the sine of the strong phase of $P / T$ and can be used as a phenomenological check of the computation of $P / T$. Figure 3 displays how a measurement of $S_{\pi \pi}$ constrains $(\bar{\rho}, \bar{\eta})$, when $P / T$ is computed in the QCD factorization approach 12]. The Figure illustrates that even if theoretical (or experimental) uncertainties prevent an accurate determination of $\sin (2 \alpha)$ in this way, the inaccurate result on $\sin (2 \alpha)$ still translates into a useful constraint in the $(\bar{\rho}, \bar{\eta})$ plane. This reflects the fact that other observables do not currently constrain $\sin (2 \alpha)$ very well.

\section{CONCLUSION}

Interpreting the new data on exclusive twobody decays of $B$ mesons in terms of fundamental parameters of the Standard Model requires - at least for the present - control over strong interaction effects. QCD-improved factorization allows us to formulate and discuss the problem 
systematically in the expansion parameters $\alpha_{s}$ and $\Lambda_{\mathrm{QCD}} / m_{b}$. The first comparisons of the theory with data on heavy-light and charmless final states are encouraging, but the theory may not always be accurate or useful. More data and calculations should help understanding where the theory works or fails, and if it fails, why it does. So far no unexpected failures are observed. If the estimates of theoretical uncertainties are correct, $B \rightarrow \pi \pi, \pi K$ together with $\left|V_{u b}\right|$ from semileptonic decay establishes $\gamma \neq 0$ at the 2-3 $\sigma$ level.

\section{ACKNOWLEDGEMENT}

I wish to thank the organizers for their generous support and hospitality and V.M. Braun and M. Diehl for comments on the manuscript.

\section{APPENDIX}

In this appendix I address a subtle point in the parameterization of infrared sensitive twist3 corrections to non-leptonic decays. The point concerns the legitimacy of integrating by parts in convolution integrals with apparently divergent boundary terms. Depending on the procedure the result appears to be either a linear or a logarithmic infrared divergence. The difference is crucial, since a linear divergence compensates the $1 / m_{b^{-}}$ suppression and hence spoils factorization even in the heavy quark limit, while a logarithmic divergence does not. The results of the following discussion have been used but have not been explained in our previous work [12].

In order to dispose of the need to introduce $B$ meson distribution amplitudes I consider an analogous problem that appears in the calculation of the pion form factor $\left\langle\pi^{+}\left(p^{\prime}\right)\left|\bar{u} \gamma_{\mu} u\right| \pi^{+}(p)\right\rangle$ at large momentum transfer $Q^{2}=-\left(p^{\prime}-p\right)^{2}$. The light-cone expansion in $1 / Q^{2}$ can be implemented by multiplying the on-shell four-quark scattering amplitude with a projection operator. For the following discussion it will suffice to restrict the projection to the two-particle quark-antiquark state and to neglect meson mass corrections. The coordinate space definitions [36]

$$
\left\langle\pi^{+}\left(p^{\prime}\right)\left|\bar{u}_{\alpha}(y) d_{\beta}(x)\right| 0\right\rangle=\frac{i f_{\pi}}{4} \int_{0}^{1} d u e^{i\left(u p^{\prime} \cdot y+\bar{u} p^{\prime} \cdot x\right)}
$$

$\times\left\{\not{ }^{\prime} \gamma_{5} \phi(u)-\mu_{\pi} \gamma_{5}\left(\phi_{p}(u)-\sigma_{\mu \nu} p^{\prime \mu} z^{\nu} \frac{\phi_{\sigma}(u)}{6}\right)\right\}_{\beta \alpha}$ are equivalent to projecting the scattering amplitude with

$$
\begin{aligned}
& M_{\beta \alpha}=\frac{i f_{\pi}}{4}\left\{\not p^{\prime} \gamma_{5} \phi(u)-\mu_{\pi} \gamma_{5}\left(\phi_{p}(u)\right.\right. \\
& \left.\left.+i \sigma_{\mu \nu} p^{\prime \mu} \frac{\phi_{\sigma}(u)}{6} \frac{\partial}{\partial k_{\nu}}\right)\right\}_{\beta \alpha}
\end{aligned}
$$

and to integrate over $u$ from 0 to 1 . (All quarkantiquark operators are assumed to be colour singlets.) I will refer to this projection as "P1". Here $k$ is the momentum of the quark in the pion and the antiquark momentum is $p^{\prime}-k$. The derivative can be decomposed into a transverse derivative, a term proportional to $p_{\nu}^{\prime}$, which does not contribute, and a term proportional to $p_{\nu}$. Using $u=p \cdot k / p^{\prime} \cdot p$ one performs an integration by parts on this term and neglecting boundary terms (because $\phi_{\sigma}(u)$ vanishes at 0 and 1), one obtains the projection "P2":

$$
\begin{aligned}
& M_{\beta \alpha}=\frac{i f_{\pi}}{4}\left\{\not p^{\prime} \gamma_{5} \phi(u)-\mu_{\pi} \gamma_{5}\left(\phi_{p}(u)\right.\right. \\
& \left.\left.-i \sigma_{\mu \nu} \frac{p^{\prime \mu} p^{\nu}}{p^{\prime} \cdot p} \frac{\phi_{\sigma}^{\prime}(u)}{6}+i \sigma_{\mu \nu} p^{\prime \mu} \frac{\phi_{\sigma}(u)}{6} \frac{\partial}{\partial k_{\perp \nu}}\right)\right\}_{\beta \alpha}
\end{aligned}
$$

The calculation of the form factor then gives

$$
\begin{array}{r}
\left\langle\pi^{+}\left(p^{\prime}\right)\left|\bar{u} \gamma_{\mu} u\right| \pi^{+}(p)\right\rangle=\frac{4 g_{s}^{2} f_{\pi}^{2}}{9 Q^{2}} \int_{0}^{1} d u d v \\
\times\left\{\frac{\phi(u) \phi(v)}{2 \bar{u} \bar{v}}\left(p+p^{\prime}\right)_{\mu}+\frac{\mu_{\pi}^{2}}{Q^{2}} T_{4}\right\},
\end{array}
$$

where the first term in the bracket represents the leading-twist result and $T_{4}$ a twist- 4 correction, which is the quantity of interest here.

The explicit form of $T_{4}$ depends on whether P1 or P2 is used for the calculation. With $\mathrm{P} 1$ the result is

$$
\begin{aligned}
\phi_{p}(u)[\{ & \left.-\frac{1}{\bar{u} \bar{v}} \phi_{p}(v)+\frac{1}{\bar{u} \bar{v}^{2}} \frac{\phi_{\sigma}(v)}{6}\right\} p_{\mu} \\
& \left.+\left\{-\frac{1}{\bar{u} \bar{v}^{2}} \phi_{p}(v)-\frac{2}{\bar{u} \bar{v}^{3}} \frac{\phi_{\sigma}(v)}{6}\right\} p_{\mu}^{\prime}\right] \\
& +\left(u \leftrightarrow v, p \leftrightarrow p^{\prime}\right),
\end{aligned}
$$

while using $\mathrm{P} 2$ leads to

$\phi_{p}(u)\left[\left\{-\frac{1}{\bar{u} \bar{v}} \phi_{p}(v)+\frac{1}{\bar{u} \bar{v}} \frac{\phi_{\sigma}^{\prime}(v)}{6}+\frac{2}{\bar{u} \bar{v}^{2}} \frac{\phi_{\sigma}(v)}{6}\right\} p_{\mu}\right.$ 


$$
\begin{aligned}
& \left.+\left\{-\frac{1}{\bar{u} \bar{v}^{2}} \phi_{p}(v)+\frac{1}{\bar{u} \bar{v}^{2}} \frac{\phi_{\sigma}^{\prime}(v)}{6}\right\} p_{\mu}^{\prime}\right] \\
& +\left(u \leftrightarrow v, p \leftrightarrow p^{\prime}\right) .
\end{aligned}
$$

The two results are identical, when an integration by parts is performed and when the boundary terms are zero. This does not seem to be justified, however, since the boundary terms are non-zero, in fact infinite, when the asymptotic distribution amplitudes $\phi_{p}(w)=1, \phi_{\sigma}(w)=6 w \bar{w}$ are inserted. Not performing the integration by parts, it is seen that the result from P1 is linearly divergent as $v \rightarrow 1$, while the result from P2 exhibits only a logarithmic divergence. Since non-perturbative infrared effects regulate the divergence at $\bar{v} \approx \Lambda / Q$ (when the quark momentum becomes of order $\Lambda$ ), the P1-result is of or$\operatorname{der} Q / \Lambda$, parametrically larger than the $\mathrm{P} 2$-result which is only of order $\ln Q / \Lambda$. This can be made more explicit by regulating the convolution integrals through introducing a gluon mass $\lambda$. The main effect of this is to replace $\bar{u} \bar{v}$ by $\bar{u} \bar{v}+\xi$ where $\xi=\lambda^{2} / Q^{2}$. The convolution integrals can then be done with the two results:

$$
\begin{aligned}
& \int_{0}^{1} d u d v T_{4}(P 1)=\left(-\frac{1}{\xi}-2 \operatorname{Li}_{2}\left(-\frac{1}{\xi}\right)\right)\left(p+p^{\prime}\right)_{\mu} \\
& \int_{0}^{1} d u d v T_{4}(P 2)=-2 \operatorname{Li}_{2}\left(-\frac{1}{\xi}\right)\left(p+p^{\prime}\right)_{\mu}
\end{aligned}
$$

The difference $-1 / \xi$ can be attributed to a linearly divergent boundary term

$\lim _{\bar{v} \rightarrow 0} \frac{1}{\bar{v}(\bar{u} \bar{v}+\xi)} \frac{\phi_{\sigma}(v)}{6}=\frac{1}{\xi}$.

Now it is clear that was has gone wrong here is that one divides 0 by 0 . The numerator is $\phi_{\sigma}(1)=0$, which is always correct, but the denominator zero arises from the virtuality of a quark propagator, which approaches zero and which is not regularized by introducing a gluon mass. To make the calculation well-defined all small-virtuality regions must be properly regularized. One possibility is to assign a small mass to

\footnotetext{
${ }^{1}$ In the present approximation that neglects quark-antiquark-gluon amplitudes the asymptotic distribution amplitudes are exact and follow from the equation of motion constraints. A possible caveat here is that the solutions given above also involve an integration by parts in which boundary terms should be zero even when the equation of motion is inserted into any Green function. The following discussion shows that this assumption can be made.
}

the quarks as well. Denoting the corresponding parameter by $\eta$, this leads to the boundary term

$\lim _{\bar{v} \rightarrow 0} \frac{1}{(\bar{v}+\eta)(\bar{u} \bar{v}+\xi)} \frac{\phi_{\sigma}(v)}{6}=0$.

It is not difficult to see that in this particular case the amplitude has a finite limit for $\eta \rightarrow 0$ (but $\xi$ still non-zero) after the convolution integrals are performed. The only effect of the complete regularization is therefore to make all integrations by parts well-defined and to eliminate the spurious linearly divergent boundary term. We can therefore conclude that the correct result is

$T_{4}(P 2)=\frac{2}{\bar{u} \bar{v}}\left(p+p^{\prime}\right)_{\mu}$,

which exhibits a double logarithmic divergence, one from each convolution integral. This coincides with the result for the chirally-enhanced twist- 4 corrections obtained long ago by Geshkenbein and Terentev [37].

The discussion can be analogously repeated for non-leptonic $B$ meson decays, where the problem appears in the computation of $1 / m_{b}$-suppressed terms. The conclusion is again that integration by parts is allowed and that the correct result is obtained directly with the projector P2. On the other hand using P1 requires one to regularize carefully all propagators that can go close to mass-shell near the endpoints of the integration region.

There is a deeper reason why the infrared divergence in the twist- 4 correction to the pion form factor should not be power-like. There exist two basic scattering mechanisms that contribute to the form factor. One is the hard scattering mechanism, in which all components of a given Fock state of the pion participate in a hard interaction. The other is a soft-overlap contribution, in which case a number of Fock components with small longitudinal momentum fraction does not participate in a hard interaction. A complete asymptotic expansion of the form factor should combine both mechanisms consistently. Recent work on the analogous problem for the $B \rightarrow \pi$ form factor [5] together with the structure of the radiatively corrected QCD sum rule for the $B \rightarrow \pi$ form factor [38] suggests the following factorization formula that incorporates the hard scattering and soft-overlap contribution:

$F\left(q^{2}\right)=\frac{1}{Q^{2}} T_{2} * \phi_{2} * \phi_{2}$ 


$$
+C \xi\left(q^{2}, \mu\right)+\frac{1}{Q^{4}} T_{4}^{\mathrm{reg}}(\mu) * \phi_{3} * \phi_{3}+O\left(1 / Q^{6}\right)
$$

Here $\phi_{2}$ and $\phi_{3}$ represent twist-2 and twist-3 light-cone distribution amplitudes and $\xi\left(Q^{2}, \mu\right)$ a soft-overlap form factor which scales as $1 / Q^{4}$ and which can be defined in an effective theory in which all hard scatterings are integrated out. $T_{4}^{\text {reg }}(\mu)$ denotes the hard-scattering kernel at the twist-4 level discussed above except that the endpoint divergence is factorized into the soft-overlap form factor, such that both terms depend on a factorization parameter $\mu$. If this (conjectured) factorization formula is to be correct, the endpoint contribution of the twist- 4 term can be at most logarithmically enhanced.

\section{REFERENCES}

1. M. Beneke, G. Buchalla, M. Neubert and C. T. Sachrajda, Phys. Rev. Lett. 83, 1914 (1999).

2. M. Beneke, G. Buchalla, M. Neubert and C. T. Sachrajda, Nucl. Phys. B 591, 313 (2000).

3. A. G. Grozin and M. Neubert, Phys. Rev. D 55, 272 (1997).

4. G. P. Korchemsky, D. Pirjol and T. M. Yan, Phys. Rev. D 61 (2000) 114510.

5. M. Beneke and T. Feldmann, Nucl. Phys. B 592 (2001) 3.

6. H. Kawamura, J. Kodaira, C. F. Qiao and K. Tanaka, Phys. Lett. B 523 (2001) 111.

7. G. P. Lepage and S. J. Brodsky, Phys. Rev. D 22 (1980) 2157.

8. V. L. Chernyak and I. R. Zhitnitsky, Nucl. Phys. B 345 (1990) 137.

9. C. W. Bauer, D. Pirjol and I. W. Stewart, Phys. Rev. Lett. 87 (2001) 201806.

10. C. W. Bauer, D. Pirjol and I. W. Stewart, hep-ph/0109045.

11. A. Khodjamirian, Nucl. Phys. B 605 (2001) 558.

12. M. Beneke, G. Buchalla, M. Neubert and C. T. Sachrajda, Nucl. Phys. B 606 (2001) 245.

13. T. Muta, A. Sugamoto, M. Z. Yang and Y. D. Yang, Phys. Rev. D 62 (2000) 094020.
14. D. s. Du, D. s. Yang and G. h. Zhu, Phys. Lett. B 488 (2000) 46.

15. D. s. Du, H. u. Gong, J. f. Sun, D. s. Yang and G. h. Zhu, hep-ph/0108141.

16. M. Z. Yang and Y. D. Yang, Phys. Rev. D 62 (2000) 114019.

17. H. Y. Cheng and K. C. Yang, Phys. Rev. D 64 (2001) 074004.

18. H. Y. Cheng and K. C. Yang, Phys. Lett. B 511 (2001) 40.

19. J. Chay, Phys. Lett. B 476 (2000) 339.

20. M. Neubert and A. A. Petrov, Phys. Lett. B 519 (2001) 50.

21. M. Diehl and G. Hiller, JHEP 0106 (2001) 067.

22. J. Chay and C. Kim, hep-ph/0009244.

23. H. Y. Cheng and K. C. Yang, Phys. Rev. D 63 (2001) 074011.

24. X. S. Nguyen and X. Y. Pham, hep $\mathrm{ph} / 0110284$.

25. H. Y. Cheng, Y. Y. Keum and K. C. Yang, hep-ph/0111094.

26. M. Beneke, T. Feldmann and D. Seidel, Nucl. Phys. B 612 (2001) 25.

27. S. W. Bosch and G. Buchalla, Nucl. Phys. B 621 (2002) 459.

28. A. Ali and A. Y. Parkhomenko, hep$\mathrm{ph} / 0105302$.

29. A. L. Kagan and M. Neubert, hep$\mathrm{ph} / 0110078$.

30. X. G. He, J. Y. Leou and J. Q. Shi, Phys. Rev. D 64 (2001) 094018.

31. D. K. Ghosh, X. G. He, B. H. McKellar and J. Q. Shi, hep-ph/0111106.

32. M. Wirbel, B. Stech and M. Bauer, Z. Phys. C 29 (1985) 637.

33. See the review by H.-n. Li in these proceedings [hep-ph/0110365].

34. S. Descotes-Genon and C. T. Sachrajda, hep$\mathrm{ph} / 0109260$.

35. A. Höcker, H. Lacker, S. Laplace and F. Le Diberder, Eur. Phys. J. C 21, 225 (2001).

36. V. M. Braun and I. E. Filyanov, Z. Phys. C 48 (1990) 239.

37. B. V. Geshkenbein and M. V. Terentev, Phys. Lett. B 117 (1982) 243.

38. E. Bagan, P. Ball and V. M. Braun, Phys. Lett. B 417 (1998) 154. 